\title{
ESTUDIO DE FORMULACIONES CERÁMICAS PARA AISLADORES ELÉCTRICOS
}

\author{
Víctor Tejada ${ }^{a}$, Dominique Plée ${ }^{* b}$
}

\begin{abstract}
RESUMEN
La empresa peruana Cerartec S.A ha elaborado aisladores eléctricos cerámicos para usos en líneas de tensión media. Estos productos, tipo carrete ANSI 53-2 ("Spool and Guy Strain Insulators"), llevan mayormente materias primas de origen nacional.

El protocolo de elaboración y la formulación afectan la porosidad residual. Los factores relevantes, tanto de formulación como de proceso, han sido determinados. En el rango estudiado, existe una relación lineal entre el módulo de rotura en flexión y la porosidad. Se comprobó también la resistencia dieléctrica de los aisladores en seco y húmedo.
\end{abstract}

Palabras claves: Aisladores eléctricos, cerámicas, tensión eléctrica, resistencia dieléctrica

\section{STUDY OF CERAMIC FORMULATIONS FOR ELECTRICAL INSULATORS}

\begin{abstract}
The Peruvian company Cerartec S.A has developed ceramic-based electrical insulators for medium voltage lines. These products, ANSI 53-2 type (Spool and Guy Strain Insulators), are composed mainly of peruvian raw materials.

Both formulation and process have an influence on residual porosities. Relevant parameters of formulation and process have been identified. In the investigated range, a linear relationship was drawn between porosities and flexural modulus of rupture.

The new ceramic electrical insulators have been tested in terms of dielectric resistance, in dry and wet conditions.
\end{abstract}

Key words: Electrical insulators, ceramics, electrical voltage, dielectric strength

\section{INTRODUCCIÓN}

En el Perú, hay una demanda creciente de aisladores eléctricos, tanto para las zonas cercanas a mar, como de grandes alturas y selva. Los requisitos técnicos son muy diferentes, puesto

a. Cerartec SA, Av. Paso de los Andes Nํo 351 Of. 4 - Pueblo Libre - Lima, Perú (cerartec@cerartec.com.pe)

b. Jirón Francisco de Orellana, 206, Surco - Lima, Perú 
que cerca del mar, hay atmósferas salinas y fuerte irradiación solar. En zonas alto-andinas se encuentra un clima con cambios de temperatura bruscos, importante irradiación solar y fuertes precipitaciones, y en la selva, humedad y temperatura altas durante todo el año. Por ello, se utilizan aisladores cerámicos, de vidrio o de resina (siliconas) en función a las condiciones encontradas. Los de resina resisten bien los UV pero los pájaros de la selva los atacan. Su ventaja es su menor peso y su menor tendencia a absorber agua. Los de cerámica son resistentes a los UV, pájaros y resisten mejor a variaciones fuertes de condiciones ambientales. Los de vidrio ya no se emplean mucho por su fragilidad.

Existe una tendencia a utilizar aisladores compuestos (cerámica-resina) que combinan ventajas de ambos tipos ${ }^{1}$.

Los aisladores están normalizados en todo el mundo bajo estándares ANSI (American National Standard Institute).

Los aisladores son cerámicas sobre base de arcillas (caolinita), feldespato, cuarzo o alúmina. Contienen, después del tratamiento térmico, un residuo de cuarzo cuyo contenido disminuye con la temperatura de calcinación, mullita y una fase vítrea cuya fracción en volumen ha de ser lo suficiente para garantizar la cohesión de las partículas y eliminar la porosidad. En general, estas porcelanas cerámicas deben llevar un mínimo de $10 \%$ en volumen de fase vítrea $^{2}$. Esta controla densificación y porosidad, así como la distribución de fases e incide en las propiedades mecánicas y dieléctricas.

Las reacciones de descomposición en temperatura de la caolinita son las siguientes:

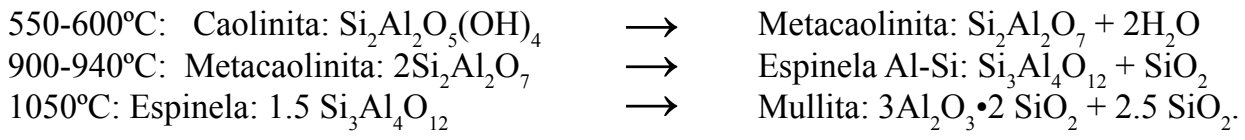

Las temperaturas exactas de reacción dependen desde luego de varios factores, tales como cristalinidad y tamaño de partículas de la caolinita. La sílice formada y la sílice inicialmente presente reaccionan con $\mathrm{Al}_{2} \mathrm{O}_{3}$ y poco a poco desaparecen a medida que se incrementa la temperatura.

Es muy conocido que la porosidad perjudica a las propiedades de cerámicas para aplicaciones eléctricas. Sangawar y col. ${ }^{3}$ aciertan aumentar la resistencia eléctrica mediante la adición de nano- $\mathrm{SiO}_{2}$ a $1 \%$ en mezclas de $\mathrm{MgO}(2 \%)$ y $\mathrm{Al}_{2} \mathrm{O}_{3}(97 \%)$. Se supone que la sílice pirogénica cuyas partículas son de muy pequeño tamaño llena la porosidad entre las partículas de $\mathrm{MgO}$ y de $\mathrm{Al}_{2} \mathrm{O}_{3}$ pero su rol es también una mayor rapidez de disolución y reacción. El tamaño de poros y el diseño del aislador influyen también sobre los resultados según Gerson ${ }^{4}$.

La ausencia de alcalinos y la eliminación de óxidos de Fe y/o Ti mejoran la resistencia dieléctrica $^{5}$. El añadido de $\mathrm{Ba}$ y $\mathrm{Zr}$ permite mejorar también la resistencia dieléctrica. En cuanto al cuarzo, el tamaño óptimo sería 10 a 30 micras, según Olupot ${ }^{6}$. Un tamaño 
demasiado pequeño lleva a mucha disolución y a una matriz vítrea perjudicial. Si son gruesas, las partículas son nudos de formación de fracturas, debido a la transformación: $\beta$-cuarzo $=>$ $\alpha$-cuarzo durante la etapa de enfriamiento. Generalmente, un contenido importante de matriz vítrea es perjudicial a altas resistencias dieléctricas.

Además, Olupot ${ }^{6}$ muestra que el óxido $\mathrm{Fe}_{2} \mathrm{O}_{3}$ se reduce durante la calcinación, liberando oxigeno según la ecuación: $3 \mathrm{Fe}_{2} \mathrm{O}_{3}=2 \mathrm{Fe}_{2} \mathrm{O}_{3}+0,5 \mathrm{O}_{2}$. Eso influye de manera negativa sobre la resistencia mecánica debido a la generación de poros. En el sistema que estudió (cuarzo, arcilla, feldespato), el módulo de rotura, la densidad y la fuerza dieléctrica son óptimos a $1250^{\circ} \mathrm{C}-1270^{\circ} \mathrm{C}$. A más alta temperatura, las propiedades disminuyen porque la fase vítrea aumenta a expensas del cuarzo ${ }^{7}$.

El rol del cuarzo es mejorar las propiedades mecánicas en "crudo" pero hace perder plasticidad, por lo que se debe acotar precisamente su contenido. Las arcillas brindan la cohesión. La alúmina da refractaridad y el feldespato actúa como fundente.

La patente US 3,674,519 $9^{8}$ explica que la dureza es óptima con menos de $30 \%$ de cuarzo y que $>97 \%$ de las partículas deben medir menos de 50 micras, siendo la temperatura de sinterización entre $1200^{\circ} \mathrm{C}$ y $1350^{\circ} \mathrm{C}$.

Esta conclusión ha sido confirmada cualitativamente por otros autores. Los granos de cuarzo incrustados en la matriz perjudican a la resistencia por la ocurrencia de micro grietas causadas por la inversión $\beta-\alpha$ durante el enfriamiento ${ }^{9}$. Por consiguiente, varios estudios trataron de remplazar el cuarzo por otros materiales, como kianita ${ }^{9}$, alúmina ${ }^{10}$, cáscara de $\operatorname{arroz}^{11}$, sillimanita ${ }^{12}$.

La influencia del feldespato sobre propiedades mecánicas ha sido estudiada; el uso de feldespatos sódicos en lugar de feldespatos potásicos es beneficioso ${ }^{13}$.

La fuerza dieléctrica depende también de factores menos controlados y a veces imprevistos. Por ejemplo, la aplicación simultánea de radiaciones energéticas, campo eléctrico y temperaturas altas durante un periodo de tiempo suficiente induce efectos permanentes, tales como rotura dieléctrica a más baja tensión, lo que constituye un problema de suma importancia en reactores de fusión nuclear ${ }^{14}$.

A partir de materias primas de Nigeria, Anih pudo obtener una fuerza dieléctrica de $26 \mathrm{kV}$ con la formulación: $10 \%$ de arcilla, $30 \%$ de caolinita, $22 \%$ de feldespato y $38 \%$ de cuarzo. El proceso era en vía seca (presión de 2000 bars) ${ }^{15}$.

La evaluación de la porosidad se puede realizar de forma no destructiva, mediante el uso de ultrasonidos como lo demuestran Fleszyński y col. ${ }^{16}$. De ello, se pueden calcular los módulos de Poisson y el de Young.

Por fin, cabe recalcar el hecho que, debido a la fuerte demanda de aisladores eléctricos en los 
últimos años por el crecimiento de las industrias de distribución de energía, la disponibilidad de aisladores en fin de vida aumentó drásticamente, en millones de toneladas en el mundo, lo que representa un problema. Es factible la transformación de estos desechos en otros productos, tales como aislantes térmicos ${ }^{17}$. Para lograr las condiciones de alta performance y la menor dispersión de calidad de las propiedades, se requiere temperaturas de no más de $1300{ }^{\circ} \mathrm{C}$ y un buen control de las condiciones térmicas (temperatura homogénea, velocidad de calentamiento, velocidad de enfriamiento, atmósfera y ciclo de quema) por lo que requiere quemadores con control de ingreso de gas y aire.

\section{PARTE EXPERIMENTAL}

Los aisladores tipo 53-2, cuyo proceso de preparación se trató de estudiar, son normalmente diseñados para $25 \mathrm{kV}$ en seco y $12 \mathrm{kV}$ en húmedo, posición vertical [Norma ANSI C 29.3]. Para fabricar los aisladores, se elaboraron moldes de acero para moldear la masa precursora del aislador. El proceso también consta de una etapa final de esmaltado para sellar toda porosidad residual después del moldeo y secado y una etapa final de sinterización a 1250$1300^{\circ} \mathrm{C}$.

\section{Formulación}

La preparación de los aisladores se realiza por mezcla de arcillas, feldespato, cuarzo y alúmina con la debida cantidad de agua. Luego, se realiza un zarandeo con malla 150 micras para eliminar las partículas gruesas.

Se realiza una etapa de prensado (hasta 900 bars) y un torneado en crudo para eliminar los defectos y rectificar dimensiones.

Finalmente, se seca al aire durante 24 horas y luego, en estufa a $110^{\circ} \mathrm{C}$ durante 12 horas.

La operación siguiente consiste en un esmaltado por inmersión o "spray" y un último tratamiento térmico a $1270-1280^{\circ} \mathrm{C}$ durante 12 horas.

Los esmaltes empleados fueron formulaciones propias de Cerartec, incluyendo cuarzo, pigmentos y feldespato, como insumos principales. El pigmento marrón ( $\mathrm{Zn} \mathrm{Fe}, \mathrm{Cr}$ y Ni) lleva el código 10134 y el negro (Co, Fe, Cr y Ni), el código 3794, de la empresa Ferro (México).

Para la pre-selección de las formulaciones y parámetros de proceso, se realizaron probetas en forma de paralelepípedo, según el mismo proceso, pero sin esmaltar.

Las composiciones estudiadas se pueden posicionar en el diagrama ternario de fase $\mathrm{SiO}_{2}$ $\mathrm{Al}_{2} \mathrm{O}_{3}-\mathrm{Na}_{2} \mathrm{O}+\mathrm{K}_{2} \mathrm{O}$, presentado en la figura 1 . 


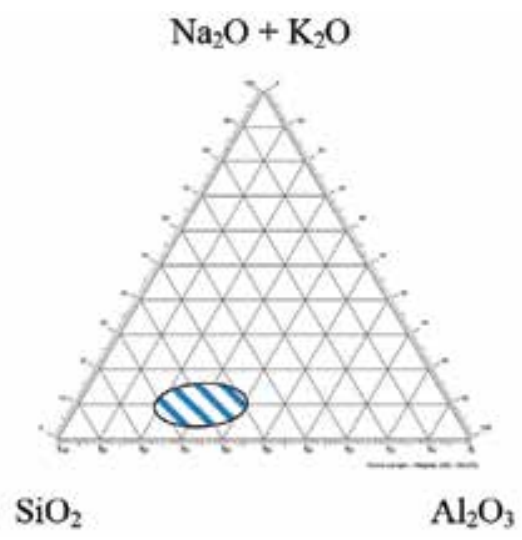

Figura 1. Diagrama de fase $\mathrm{SiO}_{2}-\mathrm{Al}_{2} \mathrm{O}_{3}-\mathrm{Na}_{2} \mathrm{O}+\mathrm{K}_{2} \mathrm{O}$ de las composiciones químicas estudiadas para la fabricación de los aisladores eléctricos.

Durante el desarrollo de la investigación, las primeras piezas salieron con leves fisuras y/o burbujas antes del esmaltado. No habría sido útil llevar a cabo las medidas de fuerza dieléctrica, pues el esmalte no es suficiente para cubrir aquellas fisuras y es conocido que la resistencia dieléctrica depende, entre otras cosas, de la porosidad del recubrimiento del aislador $^{18}$.

La dificultad de evitar fisuras y grietas radica en la difícil difusión del agua en una matriz de muy baja porosidad. Se incrementa la dificultad por los espesores diferentes en las tres dimensiones de la pieza, lo que llevó a sobredimensionar ésta y luego ajustar por torneo el precursor de aislador después del secado. Cabe señalar que hay cierta libertad en la elección de las dimensiones exactas (en rangos de algunos mm) del tipo ANSI 53-2.

Una segunda mejora se originó en fijar de manera muy precisa y repetible el parámetro de rampa de temperatura de quema, mediante un control detenido de los caudales de gas y aire.

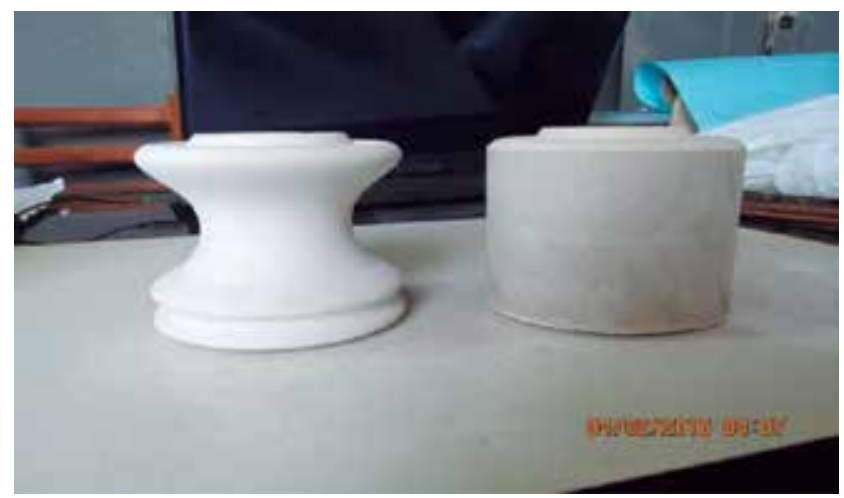

Figura 2. Fotografía del precursor del aislador tipo ANSI 53-2 después (izquierda) y antes (derecha) de tornear. 


\section{Análisis químicos y mineralógicos}

Los análisis químicos, mineralógicos y distribución granulométrica de materias primas fueron realizados por el ARPL Tecnología Industrial SA (laboratorio de Unión Andina de Cementos) por fluorescencia de rayos X (análisis químico), granulometría Laser y difracción de Rayos X (análisis mineralógico).

El equipo de fluorescencia es un BRUKER S4 Pioneer. El difractómetro es un BRUKER D4 Endeavor y se aplicó un voltaje y un amperaje respectivamente iguales a $40 \mathrm{KV}$ y $40 \mathrm{~mA}$. Se empleó la radiación $\mathrm{k} \alpha 1$ del cobre a 1,54 nm y la cuantificación de fases cristalinas se realizó mediante el método de añadidos crecientes.

La determinación de la distribución granulométrica se llevó a cabo con un equipo CILAS 1064, después de dispersar las arcillas en agua bajo ultrasonidos, durante 60 segundos, a temperatura ambiente (obscuración variable de 11 a 18\%). Se tomaron los datos de tamaño de partículas de alúmina del proveedor. Los valores indicados son el D50 en masa y la dispersión Disp., dada por la siguiente ecuación: Disp. $=($ D90 - D10 $) / D 50$, donde D10, D50, D90 = $10 \%, 50 \%, 90 \%$ de las partículas en masa están debajo del valor.

\section{Prueba de absorción de agua, densidad y choque térmico}

Para medir la absorción de agua, se realizaron ensayos de inmersión de los aisladores o probetas en agua hirviendo durante dos horas, y luego reposo por un día entero. Se pesó los aisladores o las probetas secos antes de la inmersión y se volvió a pesar después del día de reposo, trapeándolos con un tejido seco para eliminar la humedad superficial.

La porosidad abierta $\mathrm{Pa}$ se calcula entonces con las siguientes ecuaciones:

$\mathrm{M}_{\text {ag. }} / \mathrm{M}_{\text {ais }}=\%$ de agua absorbida donde $\mathrm{M}_{\mathrm{ag} .}$ es la masa de agua y $\mathrm{M}_{\text {ais }}$ la masa del aislador o probeta secos.

$\mathrm{M}_{\text {ag. }}=\mathrm{V}_{\text {ag. }} \rho_{\text {ag. }}$ donde $\mathrm{V}_{\text {ag. }}$ es el volumen de agua en la porosidad y $\rho_{\text {ag. }}$ la densidad del agua $\mathrm{M}_{\text {ais }}=\mathrm{V}_{\text {ais }} \rho_{\text {ais }}$ donde $\mathrm{V}_{\text {ais }}$ es el volumen del aislador o probeta y $\rho_{\text {ais }}$ su densidad aparente.

La porosidad $\mathrm{P}_{\mathrm{a}}=\mathrm{V}_{\mathrm{ag}} / \mathrm{V}_{\mathrm{ais}}$ y la densidad real o intrínseca del ladrillo es: $\rho_{\mathrm{i}}=\mathrm{M}_{\text {ais }} /\left(\mathrm{V}_{\mathrm{ais}}-\mathrm{V}_{\mathrm{ag}}\right)$.

Para medir la densidad, se determinó el volumen de agua desplazada al sacar el aislador después de la prueba de absorción de agua. La densidad de probetas fue determinada por la medición de sus dimensiones y masa.

Para el choque térmico, se somete un aislador a un tratamiento en agua hirviendo durante 60 minutos, seguido por un enfriamiento en una mezcla hielo/agua a $0^{\circ} \mathrm{C}$ ("Freeze-Thaw test")

\section{Pruebas mecánicas}

Las pruebas mecánicas de resistencia en flexión se realizaron con un equipo fabricado por Cerartec representado en la figura 3. Se eligió la medición de rotura en flexión, ya que es más fácil de realizar, siendo la deformación a la rotura en tracción tan pequeña que es a menudo difícil de medir. 
Los ensayos no fueron llevados a cabo sobre aisladores, sino sobre probetas (aproximadamente $15 \mathrm{~cm}$ x 2,95 cm x 0,85 cm), con las mismas formulaciones y proceso de elaboración.

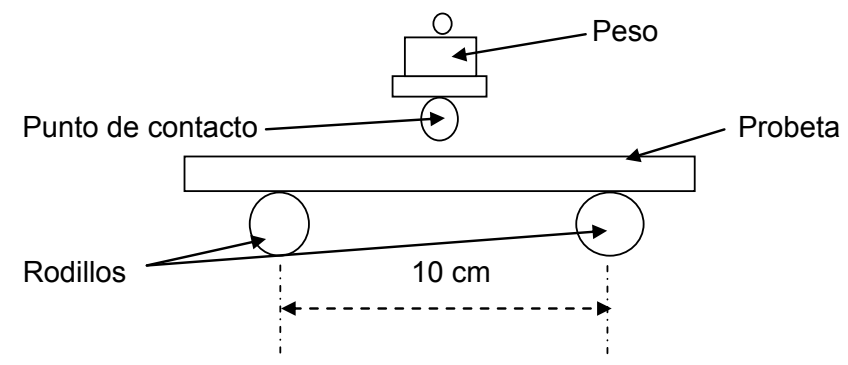

Figura 3. Equipo de medida de resistencia a la flexión. Cuenta con dos rodillos y pesos incrementales que vienen presionando la muestra. Se toma en cuenta en los cálculos la fuerza mínima que provoca la rotura.

A partir del resultado de resistencia a la flexión se calculó el módulo de rotura en flexión mediante la ecuación siguiente: $\mathrm{MR}=3 \mathrm{~F} . \mathrm{L} / 2$ b.e2 donde MR es el módulo de rotura, $\mathrm{F}$ la fuerza de rotura, L la distancia entre rodillos, b el ancho de la probeta y "e" el espesor de la misma.

\section{Ensayos de dilatometría}

Los ensayos se llevaron a cabo en el Instituto de Materiales Cerámicos de la Universidad Caxias do Sul (Brasil). El equipo usado es un Netzsch modelo DIL 402 PC y la rampa de temperatura escogida fue $5^{\circ} \mathrm{C} / \mathrm{min}$ bajo aire. El material de referencia fue alúmina.

\section{RESULTADOS Y DISCUSIÓN}

Composición mineralógica de materias primas

Tabla 1. Composición mineralógica y parámetros granulométricos de las arcillas empleadas

\begin{tabular}{|c|c|c|c|c|c|c|c|c|c|c|c|c|}
\hline \multirow[t]{2}{*}{ Arcilla } & \multicolumn{10}{|c|}{ Composición cristalográfica materias primas (\%) } & \multirow[t]{2}{*}{ D50 $(\mu)$} & \multirow[t]{2}{*}{ Disp. } \\
\hline & $\mathrm{Ca}$ & $\mathrm{Cu}$ & $\mathrm{R}$ & $\mathrm{Mu}$ & Mi & $\mathrm{T}$ & A & $\mathrm{O}$ & $\mathrm{H}$ & $\mathrm{Al}$ & & \\
\hline $\operatorname{arcilla~} \mathrm{A}$ & 49 & 51 & & & & & & & & & 4,5 & 3 \\
\hline arcilla B & 31 & 59 & 1 & 9 & & & & & & & 16,4 & 3,27 \\
\hline feldespato $\mathrm{C}$ & & 16 & & 3 & 42 & 2 & 26 & 10 & 1 & & 11,5 & 2,87 \\
\hline arcilla D & 40 & 31 & 1 & 27 & & & & & 1 & & 1 & 6,9 \\
\hline arcilla $\mathrm{E}$ & 43 & 36 & 1 & 19 & & & & & 1 & & 10 & 3,67 \\
\hline cuarzo & & 100 & & & & & & & & & 16 & 3,02 \\
\hline alúmina & & & & & & & & & & 100 & 5 & \\
\hline
\end{tabular}

$(\mathrm{Ca}=$ Caolinita, $\mathrm{Cu}=$ Cuarzo, $\mathrm{R}=$ Rutilo, $\mathrm{Mu}=$ Muscovita, $\mathrm{Mi}=$ Microclina, $\mathrm{T}=$ talco, $\mathrm{A}=$ Albita, $\mathrm{O}=$ Ortoclasa, $\mathrm{H}=$ Hematita, $\mathrm{Al}=$ Alúmina $)$. 
A parte de la arcilla D muy fina, todas las materias primas tienen un coeficiente de dispersión granulométrica en el mismo rango. Las arcillas utilizadas no son puras, pues llevan mayormente caolinita, cuarzo y muscovita. El feldespato es mayormente potásico (microclina y ortoclasa). La granulometría de la arcilla $\mathrm{D}$ es muy fina, lo que permite llenar la porosidad. Para compensar el bajo contenido de $\mathrm{Al}_{2} \mathrm{O}_{3}$ en las arcillas, fue necesario incorporar alúmina pura entre 5 y $7 \%$. La distribución granulométrica del cuarzo, con un D90 $=50$ micrones, no es óptima, según Olupot ${ }^{6}$.

\section{Características físicas y químicas de las formulaciones}

Tabla 2. Características físicas de las formulaciones de aisladores

\begin{tabular}{|l|l|l|l|l|l|l|l|}
\hline & 1 & 2 & 3 & 4 & 5 & 6 & 7 \\
\hline $\begin{array}{l}\text { Absorción de } \\
\text { agua (\%) }\end{array}$ & 1,97 & 1,4 & 1,37 & 0,58 & 1,66 & 2 & 0,9 \\
\hline $\begin{array}{l}\text { Densidad } \\
\text { aparente }\end{array}$ & 2,26 & 2,34 & 2,36 & 2,4 & 2,39 & 2,34 & 2,37 \\
\hline
\end{tabular}

La fórmula 4 parece la composición más prometedora por la baja absorción de agua, la repetibilidad de absorción de agua (no mostrada) y la plasticidad que tiene. La fórmula 7 es otra opción con baja absorción de agua y suficiente plasticidad.

Las muestras del 4 al 7 parecen tener un poquito más de densidad en promedio. Podría ser por el incremento de la arcilla D muy fina y la disminución de la arcilla A. Las fórmulas 1 y 2 no tienen lo suficiente de plasticidad. La fórmula 1 absorbe mucha agua, así como la 6 . Se solucionó el problema con el añadido de la arcilla E en la fórmula 7. Se fijó una absorción de agua límite a 1\%; por ello, las fórmulas 4 y 7 fueron elegidas.

Tabla 3. Composiciones químicas de las masas precursores de aisladores

\begin{tabular}{|l|c|c|c|c|c|c|c|}
\hline Fórmulas (\%) & $\mathbf{1}$ & $\mathbf{2}$ & $\mathbf{3}$ & $\mathbf{4}$ & $\mathbf{5}$ & $\mathbf{6}$ & $\mathbf{7}$ \\
\hline $\mathrm{SiO}_{2}$ & 66,28 & 65,64 & 66,83 & 65,11 & 64,65 & 65,67 & 64,65 \\
\hline $\mathrm{Al}_{2} \mathrm{O}_{3}$ & 22,35 & 23,1 & 22,35 & 23,32 & 23,64 & 22,25 & 22,2 \\
\hline $\mathrm{CaO}$ & 0,119 & 0,119 & 0,119 & 0,11 & 0,122 & 0,122 & 0,136 \\
\hline $\mathrm{MgO}$ & 0,161 & 0,18 & 0,169 & 0,219 & 0,225 & 0,234 & 0,246 \\
\hline $\mathrm{Fe}_{2} \mathrm{O}_{3}$ & 0,434 & 0,433 & 0,448 & 0,513 & 0,528 & 0,557 & 0,61 \\
\hline $\mathrm{Na}_{2} \mathrm{O}$ & 1,01 & 1,08 & 1,075 & 1,12 & 1,129 & 1,105 & 1,124 \\
\hline $\mathrm{K}_{2} \mathrm{O}$ & 3,15 & 3,38 & 3,347 & 3,64 & 3,7 & 3,68 & 3,86 \\
\hline $\mathrm{TiO}_{2}$ & 0,455 & 0,5 & 0,45 & 0,62 & 0,632 & 0,677 & 0,68 \\
\hline $\begin{array}{l}\text { Pérdida por } \\
\text { calcinación (\%) }\end{array}$ & 5 & 4,86 & 4,53 & 4,86 & 5 & 4,5 & 4,5 \\
\hline TOTAL & 98,9 & 99,29 & 99,32 & 99,91 & 99,91 & 98,89 & 98,03 \\
\hline
\end{tabular}


En cuanto a la suma de alcalinos y elementos de transición, la mejor fórmula es la fórmula 1 y la peor la fórmula 7. En lo que se refiere a esos parámetros, la fórmula 4 queda en el medio de la tabla. Por fin, se escogió esta y se descartó la 7 para preparar los aisladores.

Dos aisladores esmaltados, sometidos al choque térmico no mostraron desprendimiento del esmalte o fisuras, lo que hubiese ocurrido en caso de diferencias de coeficientes de expansión térmica o mala adhesión.

\section{Características mineralógicas de aisladores}

Una vez los aisladores fabricados con la fórmula 4, un poco de materia fue extraída para determinación de fases cristalinas e importancia de la fase vítrea.

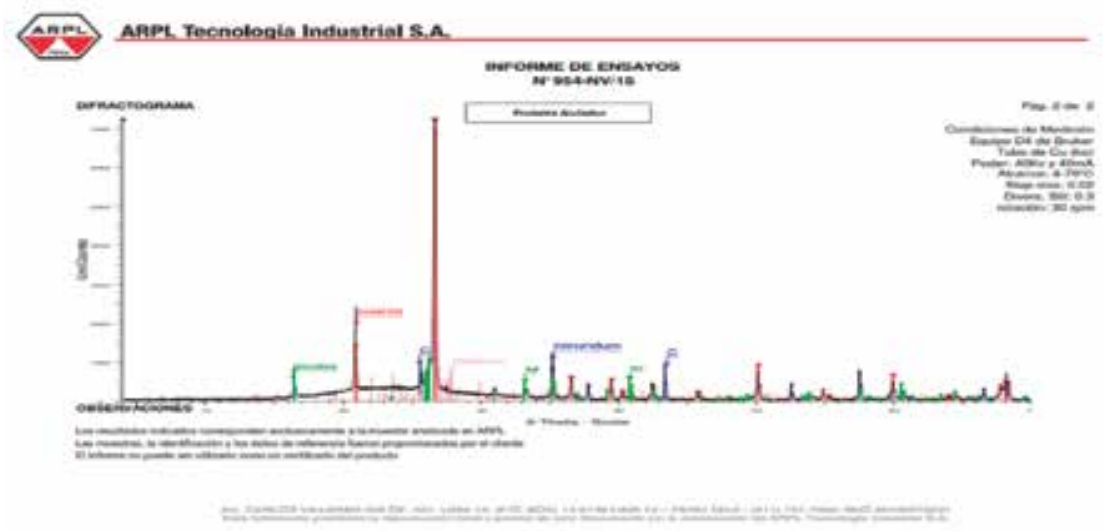

Figura 4. Espectro DRX de la fórmula 4 sinterizada

La composición mineralógica del aislador, deducida del espectro de DRX es indicada en la tabla 4, en comparación con la composición de la formulación antes de sinterizar.

Tabla 4. Composiciones mineralógicas del precursor de aislador antes y después de sinterización

\begin{tabular}{|l|c|c|}
\hline \multicolumn{1}{|c|}{ Fases } & Aislador antes de sinterizar & Aislador después de sinterizar \\
\hline Fase vítrea & 0 & 50 \\
\hline cuarzo & 36,5 & 22 \\
\hline albita & 9 & 4 \\
\hline ortoclasa & 3,5 & 9 \\
\hline microclina & 14 & 0 \\
\hline corindón & 7 & 5 \\
\hline mullita & 0 & 10 \\
\hline caolinita & 23 & 0 \\
\hline muscovita & 7 & 0 \\
\hline
\end{tabular}


Se puede notar que se reduce el cuarzo, y aun más que las cifras indicadas, ya que la transformación de la caolinita produce cuarzo y mullita. El cuarzo participa de la aparición de la fase amorfa, inducida por la presencia de feldespatos. La mullita debe su aparición a la misma reacción a partir de la caolinita. De la transformación de feldespatos, sólo la ortoclasa aumenta.

De igual manera, desaparece la muscovita que actúa como fundente a esas temperaturas.

\section{Dilatometría}

A partir de una probeta de misma composición que el aislador, y tratada al mismo momento en la estufa y horno, se llevó a cabo la prueba de dilatometría.

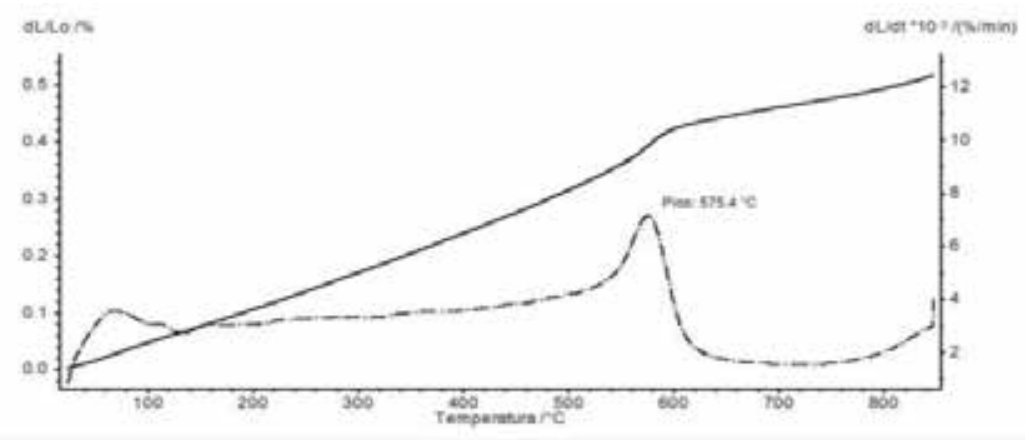

Figura 5. Curva de dilatometría de la fórmula 4

No se observa la transición de la cristobalita a $180-220^{\circ} \mathrm{C}$, dado que no se detectó esta fase por DRX. La curva muestra un accidente térmico a $575.4^{\circ} \mathrm{C}$ que corresponde a la transición del cuarzo, siendo la forma del cuarzo a temperatura ambiente, la forma $\alpha$ que cambia de manera reversible a la forma $\beta$ a $573-575^{\circ} \mathrm{C}$.

La fase $\alpha$ de baja temperatura es menos simétrica que la de alta temperatura. Asimismo, ocupa menor volumen. A pesar de que ambas fases se indexen en una red hexagonal, la forma $\alpha$ tiene una simetría trigonal mientras que la forma $\beta$ tiene la simetría hexagonal ${ }^{19}$. Esta transición se acompaña de una expansión lineal igual a $0,45 \%$. La inversión $\alpha-\beta$ del cuarzo puede causar grietas si el enfriamiento es demasiado rápido.

En el caso estudiado, la variación es pequeña, ya que el contenido residual de cuarzo es bajo y no ocasionaría fisuras por enfriamiento brusco durante el proceso de quemado.

\section{Medidas mecánicas}

El gran número de investigaciones sobre porcelanas para aisladores conllevó a tres hipótesis principales para explicar la resistencia mecánica de esas formulaciones. Carty y Senapati ${ }^{20}$ las describieron como la hipótesis de mullita, la hipótesis de refuerzo de la matriz y la hipótesis de fortalecimiento por dispersión. 
La primera sugiere que la resistencia de la porcelana depende del enclavamiento tipo "fieltro" de agujas de mullita. Cuanto más altos el contenido de mullita y enclavamiento, tanto mejor la resistencia. Los factores entonces son la temperatura de sinterización y la composición de alúmina y sílice en las materias primas.

La segunda hipótesis atañe al desarrollo de fuerzas de compresión en la fase vítrea por los diferentes coeficientes de expansión térmica de los materiales. Cuanto más importantes estas fuerzas, tanto mejor la resistencia de la porcelana. Se conoce este fenómeno como el efecto de pre-compresión.

La tercera hipótesis sustenta que las partículas dispersas en la fase vítrea, tales como cuarzo y mullita limitan el tamaño de los defectos de Griffith, lo que resulta en mejor resistencia. Existen pruebas que sustentan cada una de esas hipótesis. Entre otras cosas, los factores que influyen son los coeficientes de expansión térmica y propiedades elásticas de las fases, la fracción en volumen de las mismas y la distribución de tamaño de cristales ${ }^{20}$. La porosidad es otro factor importante.

Hay una gran cantidad de modelos que relacionan la porosidad con las propiedades mecánicas, generalmente resistencia a la tracción o compresión. Una ecuación a menudo utilizada en materiales sinterizados es la siguiente: $\sigma=\sigma 0 \exp (-\mathrm{bp})(1)$, en la cual $\sigma$ y $\sigma 0$ son las resistencias a la tracción o compresión con porosidad p y sin porosidad. La ecuación aplica en rangos limitados de porosidad ${ }^{21}$.

El modelo de la colección de granos ${ }^{22}$ expande el rango de porosidad en el cual se aplica la ecuación:

$\sigma=\sigma 0\left(1-p^{2}\right)^{2} \exp (-b p)(2)$. Se asemeja a la ecuación (1) por porosidades pequeñas.

Otra ecuación fue propuesta por Haynes [23]: $\sigma=\sigma 0(1-p) /(1+b p)(3)$.

Balshin ${ }^{24}$ propuso para cerámicas metálicas: $\sigma=\sigma 0(1-\mathrm{p})^{\mathrm{n}}(4)$ y Hasselman ${ }^{25}$, una simple relación lineal para refractarios: $\sigma=\sigma 0-\mathrm{bp}(5)$, que no puede ser correcta en todo el rango de porosidad si $b \neq \sigma_{0}$.

Aunque no hay relaciones directas entre las resistencias a la tracción y flexión, cada modelo fue probado a partir de los valores del módulo de rotura y el mejor ajuste se logró con el modelo lineal. Se trazó entonces la relación entre porosidad y módulo de rotura en flexión. 


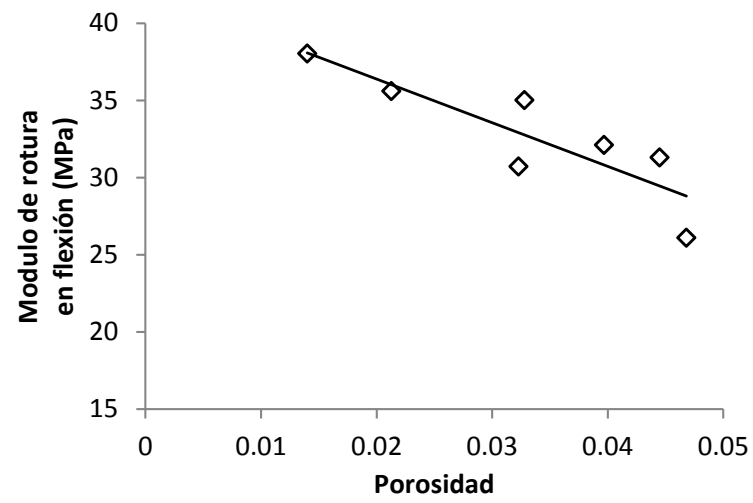

Figura 6. Relación entre módulo de rotura en flexión y porosidad

Se logra así la relación: $\mathrm{MR}=-283.44 \mathrm{p}+42.06$ donde $\mathrm{p}$ es la porosidad y MR el módulo de rotura en $\mathrm{MPa}$. El coeficiente de correlación es igual a $\mathrm{R}^{2}=0.76$, no tan bueno. Eso significa en nuestro caso que otros factores intervienen (morfología de la fase mullita y tamaño de cristales, por ejemplo), lo que es muy común en sistemas donde intervienen fenómenos químicos, tales como cristalización, disolución, transformación metamórfica, etc.

Para una porosidad nula, MR0 $=42.06 \mathrm{MPa}$, el modelo es insuficiente, pues es evidente que la linealidad deja de ser válida por una porosidad pequeña: si $\mathrm{p}=0.148, \mathrm{MR}=0$. Con más datos a porosidades más altas, uno de los otros modelos estudiados llevaría a un mejor ajuste. Por fin, los valores de módulos de rotura en flexión son lo suficientemente altos para cumplir con los criterios técnicos. Tal vez sea posible mejorar la resistencia mecánica mediante el uso de un cuarzo más fino, ya que el tamaño óptimo definido en la literatura sería 10-30 micrones.

\section{Medidas eléctricas}

Se llevaron a cabo las medidas dieléctricas en el Laboratorio $\mathrm{N}^{\circ} 6$ de Electricidad de la Universidad Nacional de Ingeniería. Dos piezas fueron probadas, según norma ANSI 29.3, en comparación con un producto importado.

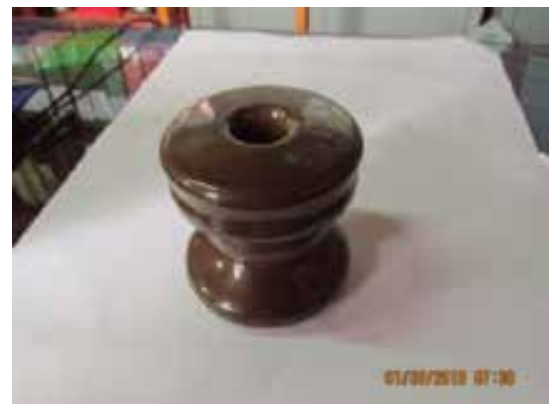

Figura 7. Aislador esmaltado probado en fuerza dieléctrica 
Los aisladores deben resistir $25 \mathrm{kV}$ y $12 \mathrm{kV}$ durante 1 minuto en seco y húmedo, respectivamente. La tabla 5 recopila los valores de voltaje y corriente de fuga para los tres productos.

Tabla 5. Voltajes y corrientes de fuga para los tres aisladores

\begin{tabular}{|l|c|c|c|}
\hline & Cerartec 1 & Cerartec 2 & Importado \\
\hline \% absorción de agua & 0,36 & 0,85 & 0,03 \\
\hline En seco & & & \\
\hline Voltaje (KV) & 25 & 22 & 25 \\
\hline Corriente de fuga (mA) & 0,23 & 0,36 & 0,31 \\
\hline En húmedo & & & \\
\hline Voltaje (kV) & 15 & No estable & No estable \\
\hline Corriente de fuga (mA) & 0,14 & No estable & No estable \\
\hline
\end{tabular}

El único producto que cumple con la norma es el producto Cerartec 1. Por ello, fue él cuyas propiedades de aislamiento fueron medidas. La medida se realizó, sometiendo la pieza a 500 V. Un multímetro indica la resistencia que en ese caso fue igual a $107 \mathrm{M} . \mathrm{Ohm}$.

Aparece claramente que la absorción de agua ejerce una influencia importante sobre los resultados, pero no es el único parámetro. Por lo tanto, se estableció, para la fórmula 4, el límite de absorción de agua posible a $<0,5 \%$. Se supone que el producto importado lleva elementos químicos poco favorables a une buena resistencia dieléctrica en húmedo.

\section{CONCLUSIÓN}

Se pudo desarrollar formulaciones de aisladores eléctricos basados en mezclas de arcillas, cuarzo, feldespato y alúmina. El proceso cuenta con etapas de moldeo, secado, calcinación, esmaltado y tratamiento térmico final.

Para algunas formulaciones, la absorción de agua es $<1 \%$ después de esmaltado y no solamente la formulación influye sobre este parámetro, sino también el proceso térmico.

Las propiedades mecánicas son suficientes para la aplicación final y dependen de la porosidad según un modelo lineal en el rango de porosidades investigado.

La curva de dilatometría muestra que queda poco cuarzo en al aislador final, tal como lo comprueba la difracción de Rayos X que, además muestra que las fases presentes son la fase vítrea, un poco de cuarzo y mullita.

Las propiedades eléctricas son buenas con la fórmula 4, cuando se respecte un límite de absorción de agua $<0,5 \%$. 


\section{AGRADECIMIENTO}

Los autores reconocen y agradecen el soporte financiero del INNOVATE Perú (FINCyT) en el marco del programa PIPEI. Título del proyecto:

Mejoramiento de aisladores eléctricos de cerámicos vitrificados para uso en sistemas de media tensión. Código: PIPEI-5-P-004-13

\section{REFERENCIAS BIBLIOGRÁFICAS}

1. Goudle J. Silicone rubber for electrical insulators. [en línea]. DOW Corning; 1998. [accesado 08 de diciembre del 2014]. Disponible en: http://www.dowcorning.com/ content/publishedlit/rubber_tech98.pdf

2. Cho YS, Yoon KH. Dielectric ceramics. En Nalwa HS. Editor. Handbook of Advanced Electronic and Photonic Materials and Devices. New York: Academic Press. 2001; 4(5): 175-199.

3. Sangawar SR, H.H. Kumar HH. Alumina-based ceramic material for high-voltage ceramic substrate. Def Sci J. 2006; 56(2): 269-278.

4. Gerson R, Marshall TC. Dielectric Breakdown of Porous Ceramics. J Appl Phys. 1959; 30(11): 1650 .

5. Roula A, Boudeghdegh K, Boufafa N. Improving usual and dielectric properties of ceramic high voltage insulators. Ceramica. 2009; 55: 206-208.

6. Olupot PW. Assessment of Ceramic Raw Materials in Uganda for Electrical Porcelain. [Tesis de licenciatura]. Stockholm: Royal Institute of Technology; 2006.

7. Olupot PW. Characterization of ceramic raw materials in Uganda for production of electrical porcelain electrical insulators. [Tesis doctoral]. Stockholm: Royal Institute of Technology; 2010.

8. Higuchi N, Ogawa Y, Watanabe A, inventores; NGK Insulators Ltd, cesionario. Method of manufacturing porcelain for electric insulators by controlling grain size. US Patent 3,674,519, 1972 Julio 4.

9. Schroeder EJ. Inexpensive high strength electrical porcelain. Am Ceram Soc Bull.1978; $57 ; 526$.

10. Kobayashi Y, Ohira O, Ohashi Y, Kato E. Strength and Weibull distribution of alumina strengthened whiteware bodies. J Ceram Soc Jpn Int Ed. 1987; 95: 837-841.

11. Prasad CS, Maiti KN, Venugopal R. Effect of rice husk ash in whiteware compositions. Ceram Int. 2001; 27: 629-635.

12. Maity S, Sarkar BK. Development of high-strength whiteware bodies. J Eur Ceram Soc. 1996; 16:1083-1088.

13. Das KS, K. Dana K. Differences in densification behaviour of K- and Na-feldsparcontaining porcelain bodies. Thermochimica Acta. 2003; 406(1-2): 199-206.

14. Chen Y, Clinard FW, Evans BD, Farnum EH, French RH, Gonzalez R, et al. Electrical breakdown of insulating ceramics in a high-radiation field. J Nucl Mater. 1994; 217(12): $32-47$.

15. Anih LU. Indigenous manufacture and characterization of electrical porcelain insulator. 
Nigerian Journal of Technology. 2005; 24(1): 4-50.

16. Fleszyński J, Ranachowski P, Ranachowski Z, Rejmund F. Ultrasonic non-destructive diagnostics of ceramic line insulators. Insight - Non-Destructive Testing and Condition Monitoring. 2005; 47(9):530-535.

17. Xua N, Lia S, Lia Y, Xuea Z, Yuanb L, Zhang J, et al. Preparation and properties of porous ceramic aggregates using electrical insulators waste. Ceram Int. 2015; 41(4): 5807-5811.

18. Pawlowski L. The relationship between structure and dielectric properties in plasmasprayed alumina coatings. Surf Coat Technol. 1988; 35(3-4): 285-298.

19. Moss GW. Mathematical Models of the Alpha-Beta Phase Transition of Quartz. PhD Thesis, , 1999. [Tesis doctoral]. Blacksburg: Virginia Polytechnic Institute; 1999.

20. Carty WM. U. Senapati U. Porcelain-raw materials, processing, phase evolution, and mechanical behavior. J Am Ceram Soc. 1998; 81(1): 3-20.

21. Hamiuddin M. Correction between mechanical properties and porosity of sintered iron and steels. Powder Metall Int. 1986; 18: 73-76.

22. Sherban NI. Poroshkovaya Metallurgiya (Powder Metallurgy). 1973; 9: 57.

23. Haynes R. Powder Metall. 1971; 14(27): 71-77.

24. Balshin MY. Relation of mechanical properties of poder metals and their porosity and the ultimate properties of porous metal-ceramic materials. DOKL Askd SSS. 1949; 67(5): 831-834.

25. Hasselman DPH. Unified theory of termal shock resistance of ceramic materials. J Am Ceram Soc. 1969; 52: 600-604. 